\title{
Hyperuricemia Causes Glomerular Hypertrophy in the Rat
}

\author{
Takahiko Nakagawa ${ }^{a}$ Marilda Mazzalia Duk-Hee Kanga J ohn Kanellis ${ }^{a}$ \\ Susumu Watanabe ${ }^{a}$ Laura G. Sanchez-Lozada ${ }^{b}$ Bernardo Rodriguez-Iturbe ${ }^{c}$ \\ J aime Herrera-Acostab Richard J . J ohnson ${ }^{a}$ \\ aDepartment of Medicine-Nephrology, Baylor College of Medicine, Houston, Tex., USA; \\ bNephrology, Instituto Nacional de Cardiología I, Mexico City, Mexico; cHospital Universitario and \\ Universidad del Zulia, Maracaibo, Venezuela
}

\section{Key Words}

Uric acid · Glomerular hypertrophy · Glomerulosclerosis

\begin{abstract}
Background/Aims: Rats with mild hyperuricemia develop systemic hypertension, interstitial renal disease, afferent arteriolopathy, and increased renin expression [Mazzali et al.: Am J Physiol 2002;6:F991-F997]. We hypothesized that hyperuricemia might also induce glomerular changes. Methods: We reviewed renal biopsies of rats previously made hyperuricemic for 7 weeks with the uricase inhibitor, oxonic acid. Controls included normal rats and oxonic acid-treated rats administered allopurinol, benziodarone, hydrochlorothiazide, or enalapril. Glomeruli were examined for size (computer image analysis) and structure (histology). An additional group of rats were administered oxonic acid or control diet for 6 months. Results: Renal biopsies showed that hyperuricemic rats had a $30 \%$ increase in glomerular tuft area $(p<0.01)$; these changes were prevented by allopurinol and benziodarone. Control of blood pressure with hydrochlorothiazide did not prevent the development of glomerular hypertrophy, whereas enalapril partially reduced the glomerular hypertrophy. Prolonged hyperuricemia was associated with the development of microal-
\end{abstract}

\section{KARGER}

Fax +41613061234

E-Mail karger@karger.ch

www.karger.com
(C) 2003 S. Karger AG, Basel

0250-8095/03/0231-0002\$19.50/0

Accessible online at:

www. karger.com/journals/ajn buminuria $(\mathrm{p}<0.05)$ and glomerulosclerosis ( 22 vs. $10 \%$, $\mathrm{p}<0.05)$ compared to control rats. Conclusions: Hyperuricemic rats develop glomerular hypertrophy that can be prevented in part by ACE inhibitor therapy. Prolonged hyperuricemia is associated with the development of glomerulosclerosis in the rat.

Copyright $\odot 2003$ S. Karger AG, Basel

\section{Introduction}

There has been controversy over the role of uric acid in renal disease [1]. It is known that the majority of patients with gout develop variable degrees of glomerulosclerosis and tubulointerstitial fibrosis, but some authorities have ascribed these changes to coexistent aging- or hypertension-related renal injury [2-4]. Interestingly, no animal model of mild hyperuricemia has been available to directly investigate the role of uric acid in the development of renal injury.

Most mammals have low serum uric acid due to the presence of uricase, a hepatic enzyme that degrades uric acid to allantoin. Humans and the great apes lack this enzyme, due to several mutations that occurred during early hominoid evolution 10-15 million years ago [5]. To develop a model of mild hyperuricemia in rats, we fed low
Takahiko Nakagawa

Department of Medicine-Nephrology

One Baylor Plaza ALKEK N520

Houston, TX 77030 (USA)

Tel. +1 713798 5835, Fax +1 713798 5010, E-Mail takahiko@bcm.tmc.edu 
doses of a uricase inhibitor (oxonic acid, OA) which resulted in mild elevations of serum uric acid without the intrarenal crystal deposition or acute renal failure that had occurred in previous models [6, 7]. Hence, this model was considered ideal for studying the effects of mild hyperuricemia on renal disease.

An interesting finding was that mildly hyperuricemic rats developed hypertension and this occurred in the presence of either a low or normal salt diet [7]. Hyperuricemic rats also developed mild renal interstitial fibrosis, with collagen deposition and macrophage infiltration [6] as well as arteriolopathy of the preglomerular vessels [7]. The renal changes were accompanied by activation of the renin angiotensin system (RAS) and by inhibition of neuronal nitric oxide synthase (NOS-1) expression in the macula densa [6, 7]. All of the renal changes could be prevented if the uric acid levels were maintained in the normal range by the use of allopurinol.

Structural glomerular changes were initially not recognized. However, changes such as glomerular hypertrophy may be difficult to note unless careful measurements are made. Glomerular hypertrophy can result from stimulation of the RAS and is associated with the development of glomerulosclerosis [8]. We therefore re-examined our original tissue [7] in a blinded manner for glomerular changes using computer image analysis. We also examined the effect of longstanding hyperuricemia on glomerular changes.

\section{Materials and Methods}

\section{Experimental Design}

All experiments were approved by the University of Washington Animal Care Committee. Details of this study have been previously described [7]. Specifically, hyperuricemia was induced in male 200250 g Sprague-Dawley rats (Simonsen Laboratories, Gilroy, Calif., USA) by treating the rats with $2 \%$ oxonic acid (OA) (Sigma, St. Louis, Mo., USA) provided in a low salt $(0.125 \% \mathrm{NaCl})$ (Ziegler Bros., Gardners, Pa., USA) diet for 7 weeks $(n=5)$. Controls included low salt $(\mathrm{LS})$ diet alone $(\mathrm{n}=5)$ or rats treated with OA/LS diet in which hyperuricemia was prevented by allopurinol (AP, $150 \mathrm{mg} / \mathrm{l}$ in the drinking water) $(\mathrm{n}=5)$ or benziodarone $(\mathrm{BZ}, 10-15 \mathrm{mg} / \mathrm{kg} / \mathrm{day}$ in the drinking water) $(n=5)$. To investigate the role of blood pressure in the renal changes, two additional groups received OA/LS diet with either hydrochlorothiazide (HCTZ) $(3 \mathrm{mg} / \mathrm{kg} /$ day in the drinking water, $\mathrm{n}=5)$ or enalapril $(1 \mathrm{mg} / \mathrm{kg} /$ day in the drinking water, $\mathrm{n}=5)$. For the HCTZ and enalapril, the concentration of drug in the drinking water was adjusted every 2 days to ensure proper dosing.

To examine the effect of sustained mild hyperuricemia on the glomerulosclerosis, OA was administered for 6 months to additional rats on an LS diet (LS + OA) and compared with control rats treated with LS diet alone $(n=4$ each $)$.

Uric Acid and Glomerular Hypertrophy
For all studies, systolic blood pressure (SBP) was measured by tail-cuff sphygmomanometer using an automated system with photoelectric sensor (IITC, Life Science) that has been shown to correlate with intra-arterial measurements [9]. All animals were preconditioned for blood pressure measurements 1 week before each experiment. Serum uric acid was measured by the carbonate phosphotungstate method [10].

\section{Renal Histology}

Renal biopsies were fixed in methyl-Carnoy's and embedded in paraffin. Four-micrometer sections of methyl-Carnoy's fixed tissue were stained with periodic acid-Schiff (PAS) reagent. Indirect immunoperoxidase staining was performed as described previously [11], with the following specific antibodies: endothelial cells with JG-12 (a gift of Dontscho Kerjaschki, University of Vienna, Austria) and collagen IV with goat anti-human collagen IV antibody (Southern Biotechnology Assoc., Inc., Birmingham, Ala., USA). To detect mesangial cell proliferation, double immunostaining was performed with an antibody to the proliferating cell nuclear antigen (PCNA) (PC 10; Cappel, Aurora, Ohio, USA), and Thy-1.1 (OX-7; Serotec, Oxford, UK). Tissue sections were first incubated with PCNA antibody overnight at $4{ }^{\circ} \mathrm{C}$, followed sequentially by biotinylated horse anti-mouse IgG serum, peroxidase-conjugated avidin $\mathrm{D}$, and color development with diaminobenzidine (DAB) with nickel chloride. After incubation in $3 \% \mathrm{H}_{2} \mathrm{O}_{2}$ for 10 min to eliminate any remaining peroxidase activity, sections were incubated with $\mathrm{OX}-7$ for $3 \mathrm{~h}$ at room temperature, followed by biotinylated horse anti-mouse $\mathrm{IgG}$ for $30 \mathrm{~min}$ at room temperature. After incubation to peroxidase-conjugated avidin D, color was developed with DAB.

\section{Quantification of Morphology}

All quantification was blinded. Glomerular hypertrophy was quantified as the glomerular tuft cross-sectional area as measured by computer image analysis. Glomerular hypertrophy in the cortex was assessed by measuring the tuft area of glomeruli in which the vascular pole was evident (using at least 30 glomeruli per biopsy). This was performed to reduce the possibility of including tangentially cut glomeruli. Glomerular injury (glomerulosclerosis) was defined as an increment in PAS-stained material associated with loss of cellular elements, collapse of capillary lumens, and amorphous hyaline material with or without adhesions to the Bowman's capsule.

Changes in endothelial density and morphologic staining were evaluated by immunostaining with JG-12 [11]. Collagen IV deposition in glomeruli was also analyzed histologically.

\section{Statistical Analysis}

Values are expressed as mean \pm SD. Differences in various parameters between groups were evaluated by ANOVA with appropriate correction for multiple comparisons (Bonferroni/Dunn or Fisher's)

\section{Results}

Glomerular Hypertrophy Develops in Hyperuricemic Rats

We initially evaluated the glomerular changes that occurred with 7 weeks of hyperuricemia utilizing renal biopsies from a previous study [7]. The blood pressure 
Fig. 1. Glomerular hypertrophy develops in hyperuricemic rats. In contrast to the normal glomerulus from a rat on a control LS diet (A), the glomerulus from a hyperuricemic rat on an LS diet was substantially larger (B). 7 weeks. PAS. $\times 400$.
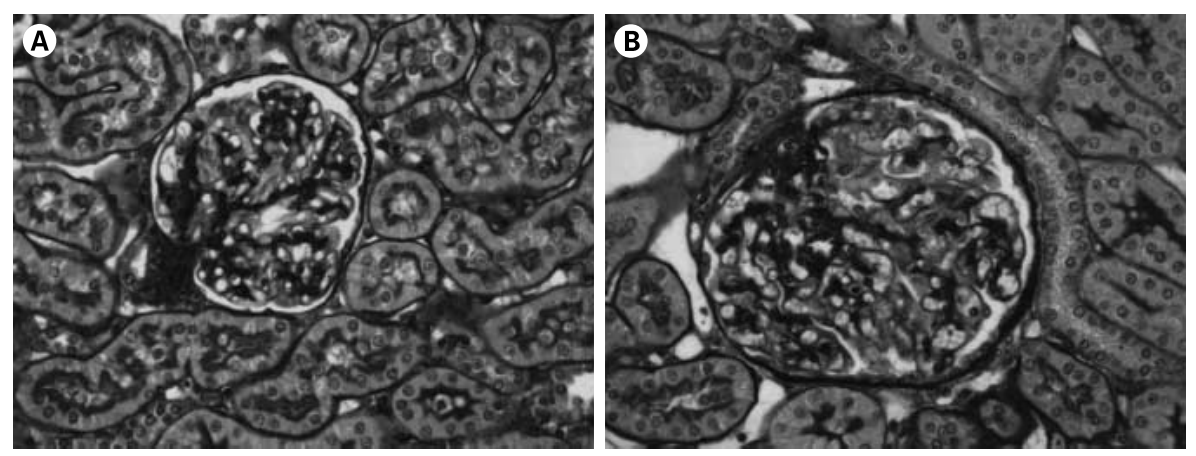

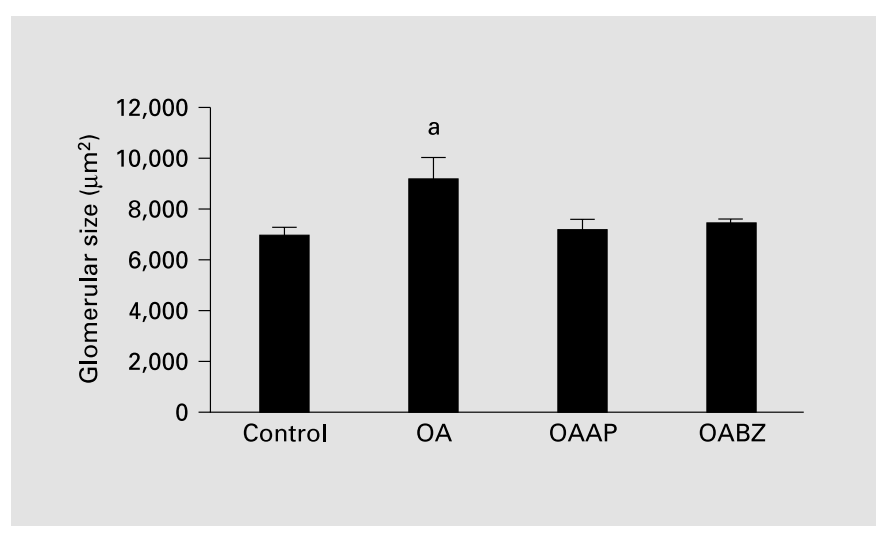

Fig. 2. Glomerular hypertrophy induced by hyperuricemia at 7 weeks. Cortical glomerular tuft area at 7 weeks was increased in hyperuricemic (OA) rats compared to controls and was blocked by treatment with allopurinol (OAAP) or benziodarone (OABZ). ${ }^{a} \mathrm{p}<$ 0.01 vs. control, OAAP, OABZ.

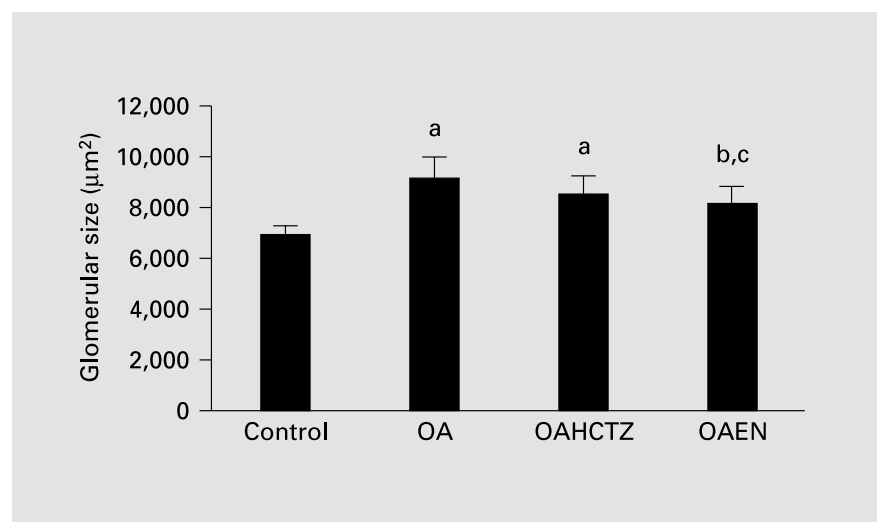

Fig. 3. Effect of controlling blood pressure on glomerular hypertrophy. Cortical glomerular tuft area was not significantly reduced by HCTZ. In contrast, enalapril reduced glomerular hypertrophy compared to hyperuricemic rats, although hypertrophy was still present when compared to controls. ${ }^{\mathrm{a}} \mathrm{p}<0.01$ vs. control, ${ }^{\mathrm{b}} \mathrm{p}<0.05$ vs. OA, ${ }^{\mathrm{c}} \mathrm{p}<0.05$ vs. control.
Table 1. Uric acid levels and blood pressures in the different groups

\begin{tabular}{lll}
\hline & $\begin{array}{l}\text { Uric acid level } \\
\mathrm{mg} / \mathrm{dl}\end{array}$ & $\begin{array}{l}\mathrm{SBP} \\
\mathrm{mm} \mathrm{Hg}\end{array}$ \\
\hline Control (LS) & $1.39 \pm 0.07$ & $126.8 \pm 4.9$ \\
Hyperuricemia (LS/OA) & $1.84 \pm 0.21^{\mathrm{a}}$ & $146.9 \pm 8.5^{\mathrm{a}}$ \\
Allopurinol (LS/OA/AP) & $1.32 \pm 0.06$ & $119.3 \pm 5.6$ \\
Benziodarone (LS/OA/BZ) & $1.40 \pm 0.13$ & $127.8 \pm 6.3$ \\
HCTZ(LS/OA/HCTZ) & $1.94 \pm 0.07^{\mathrm{a}}$ & $126.0 \pm 6.8$ \\
Enalapril (LS/OA/EN) & $1.86 \pm 0.21^{\mathrm{a}}$ & $122.5 \pm 4.5$
\end{tabular}

Data obtained from Mazzali et al. [7].

$\mathrm{EN}=$ Enalapril; $\mathrm{HCTZ}=$ hydrochlorothiazide; $\mathrm{LS}=$ low salt diet; $\mathrm{OA}=$ oxonic acid.

a $\mathrm{p}<0.05$ vs. control.

and uric acid levels from those rats and controls are shown in table 1. Structural glomerular changes were not noted in the original study; however, careful analysis using computer image methodology was not performed.

When glomerular cross sections were compared between groups using light microscopy (PAS staining), a difference in glomerular size was evident (fig. 1). Computer image analysis confirmed that the glomerular tuft area in both the cortex and juxtamedullary areas were increased (30\% greater) in the hyperuricemic rats compared to normal controls (fig. 2). Glomerular hypertrophy was not observed in OA-treated rats in which hyperuricemia was prevented with AP or BZ (fig. 2).

To determine the effect of controlling the blood pressure on the glomerular hypertrophy, we evaluated renal biopsies from hyperuricemic rats whose blood pressure was controlled with HCTZ. HCTZ treatment did not affect the level of uric acid compared to rats given OA alone although it did prevent the development of hypertension (table 1). While glomerular hypertrophy tended to 

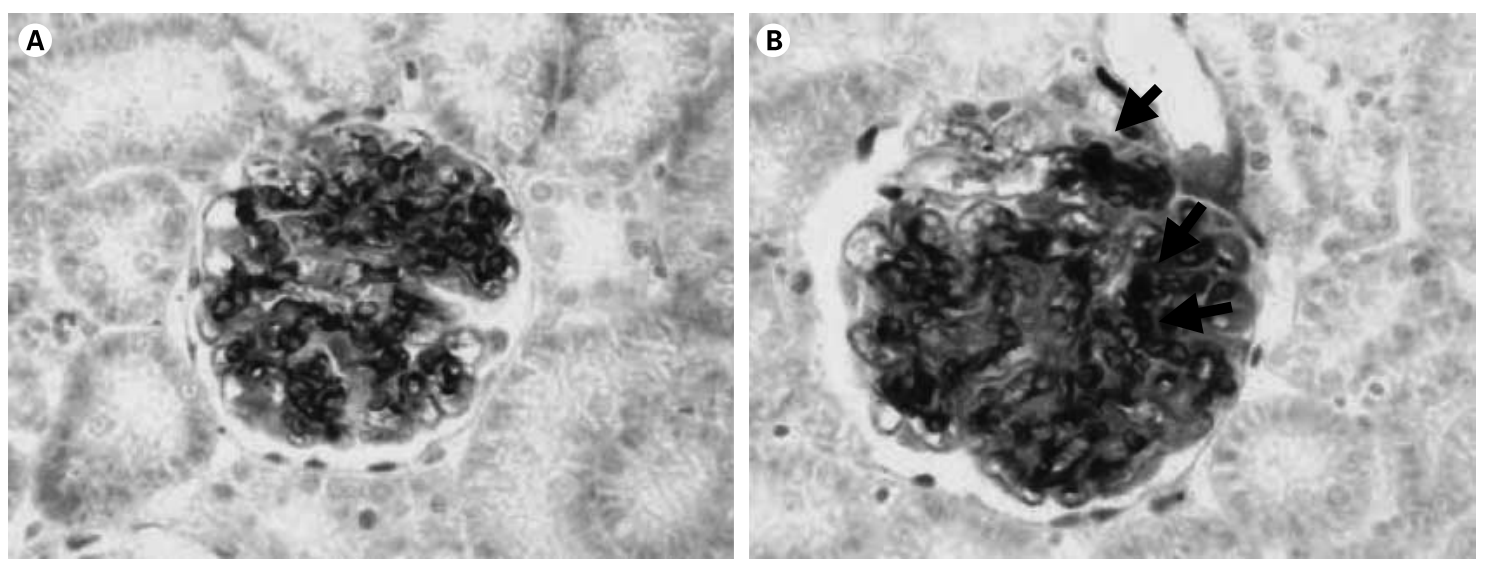

Fig. 4. Mesangial cell proliferation occurs in hyperuricemic rats. Compared to control rats at 7 weeks (A), rats with hyperuricemia showed increased mesangial cell proliferation (B), as noted by double staining of tissue sections with a mesangial cell marker (OX-7, brown cytoplasmic stain), and a cell proliferation marker (PCNA, black nuclear staining). Immunoperoxidase. $\times 400$.

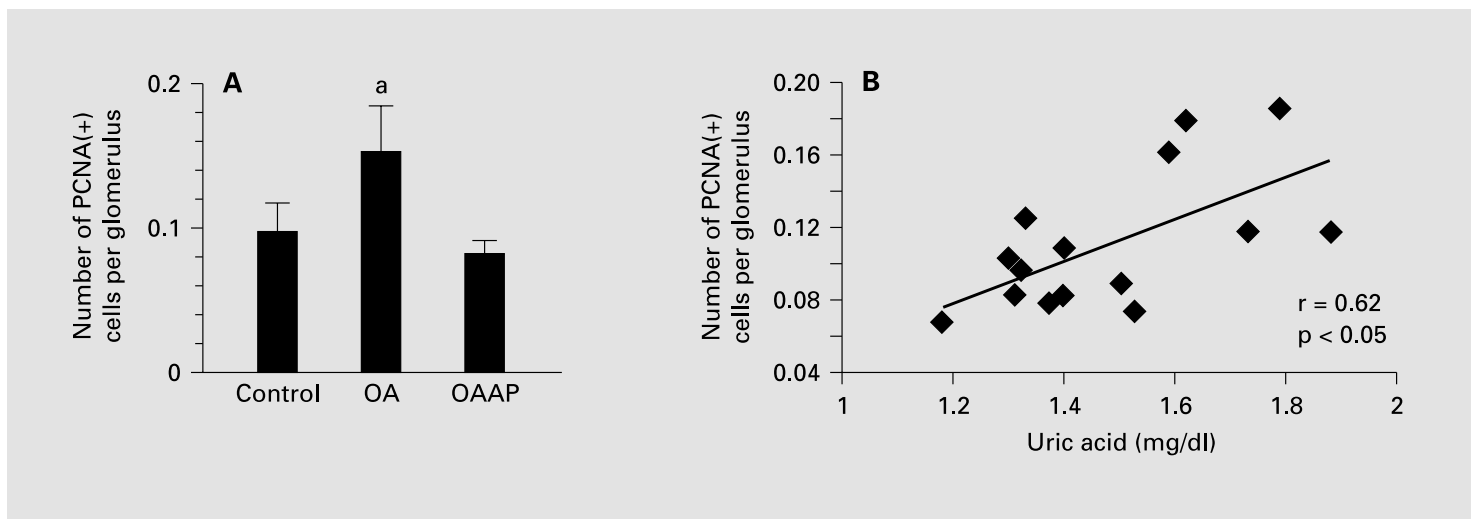

Fig. 5. Relationship of glomerular cell proliferation with hyperuricemia and glomerular hypertrophy. There is an increase in glomerular cell proliferation (PCNA+ cells/glomerular cross section) in hyperuricemic rats (OA) compared to control rats or OA rats treated with allopurinol (OAAP). A ${ }^{a} \mathrm{p}<0.05$ vs. control, OAAP. B There is a correlation between uric acid and glomerular cell proliferation.

be lower than that observed with hyperuricemia alone, it was not significant. In contrast, the glomerular tuft area remained elevated compared to control animals (fig. 3).

We also evaluated the effect of an angiotensin-converting enzyme (ACE) inhibitor, enalapril, on glomerular hypertrophy in hyperuricemic rats. Enalapril also did not affect serum uric acid levels although blood pressure was equivalently reduced to that observed with HCTZ (table 1). Unlike HCTZ, enalapril-treated rats had less glomerular hypertrophy compared to hyperuricemic controls, although it was still significantly increased compared to normal controls (fig. 3).

Uric Acid and Glomerular Hypertrophy

\section{Histologic Changes Induced by Hyperuricemia}

The glomerular hypertrophy in hyperuricemic rats was not accompanied by any detectable increase in collagen IV deposition, or any alterations in endothelial cell morphology (JG-12 stain) (data not shown). Mild mesangial cell proliferation (PCNA+/OX-7+ cells) was present in hyperuricemic rats (fig. 4), although evidence for mesangial cell activation, such as the expression of $\alpha$-smooth muscle actin, was not observed (data not shown).

An increase in total proliferating $(\mathrm{PCNA}+)$ cells within the glomerulus was shown (fig. 5A). The increase in proliferating cells $(50 \%)$ was consistent with the overall change 
in glomerular size in hyperuricemic rats. When the normouricemic (control and OA/AP) groups were compared to the hyperuricemic (OA) group, a correlation between the number of proliferating cells and serum uric acid was demonstrated $(r=0.62, p<0.05)$ (fig. 5B).

\section{Hyperuricemia Induces Glomerulosclerosis}

Glomerular hypertrophy is commonly associated with the development of glomerulosclerosis [8]. Early features of glomerulosclerosis were observed in approximately $2 \%$ of glomeruli of hyperuricemic rats at 7 weeks, but this was not significantly different from control rats (data not shown). To evaluate the long-term effects of hyperuricemia, we administered OA to rats for 6 months $(n=4)$ and compared them to diet-treated controls $(n=4)$. Serum uric acid levels were only mildly elevated in the OAtreated rats $(1.8 \pm 0.2$ vs. $1.4 \pm 0.1 \mathrm{mg} / \mathrm{dl}, \mathrm{p}<0.05)$ and final weights were not different (531.6 \pm 27.5 vs. $523.0 \pm$ $39.2 \mathrm{~g}, \mathrm{p}=\mathrm{NS})$. Hyperuricemic rats did manifest higher SBPs (144 \pm 4 vs. $123 \pm 2 \mathrm{~mm} \mathrm{Hg}$, mean for months 4-6, $\mathrm{p}<0.05)$. Hyperuricemic rats developed more albuminuria $(14.9 \pm 6.5$ vs. $8.9 \pm 2.1 \mathrm{mg} / \mathrm{dl}$ in OA vs. control, 6 months, $\mathrm{p}<0.05)$ and more glomerulosclerosis $(22 \pm 6.4$ vs. $10 \pm 4.8 \%, \mathrm{p}<0.05$, respectively).

\section{Discussion}

Rats with mild hyperuricemia develop hypertension, arteriolopathy and interstitial renal disease in the absence of intrarenal crystalline deposits $[6,7]$. In this study we examined the effect of hyperuricemia on glomerular structure. Our principal finding is that hyperuricemia induces glomerular hypertrophy that involved cortical glomeruli. The glomerular hypertrophy was not due to systemic hypertension, as HCTZ prevented hyperuricemia-induced hypertension but could not prevent glomerular hypertrophy. In contrast, inhibition of the RAS with an ACE inhibitor partially prevented glomerular hypertrophy.

Glomerular hypertrophy was measured in a blinded manner using computer image analysis. For assessing cortical glomerular size, we measured the tuft area of glomeruli in which the vascular poles were evident, as this would eliminate tangentially cut glomeruli that would falsely suggest a smaller glomerular size. Utilizing this approach, we found that glomeruli from hyperuricemic rats were $30 \%$ larger than control glomeruli. This degree of hypertrophy is similar to that observed in diabetic rats or in rats with remnant kidneys. Interestingly, we also assessed jux- tamedullary glomerular size by evaluating consecutive glomeruli at the level of the arcuate artery. We found an identical percent increase in glomerular size in juxtamedullary glomeruli in hyperuricemic rats (data not shown).

Evidence that the glomerular hypertrophy was due to the hyperuricemia was provided by the studies using AP and BZ. These agents lower uric acid either by inhibiting xanthine oxidase (AP) or by uricosuric effects (BZ). The observation that AP and BZ could prevent glomerular hypertrophy in concert with maintaining normal uric acid levels suggests that it is uric acid and not OA that is responsible for the hypertrophy.

To determine the role of SBP in the glomerular hypertrophy, we prevented the increase in blood pressure in hyperuricemic rats with HCTZ. Interestingly, HCTZ was not able to prevent hyperuricemia-induced glomerular hypertrophy. While there was some reduction in glomerular hypertrophy compared to control hyperuricemic rats, this did not reach statistical significance. This suggests that the glomerular hypertrophy is not mediated by the increase in systemic blood pressure.

Angiotensin II is an important mediator of glomerular hypertrophy [8]. Previous studies in humans have found that plasma renin levels correlate with serum uric acid in patients with essential hypertension [12], and we also found that tissue renin was increased in hyperuricemic rats [6]. In this study we showed that enalapril, an ACE inhibitor, partially reduced glomerular hypertrophy. This suggests that uric acid-induced glomerular hypertrophy is mediated by the RAS. However, since AP and BZ were more effective at reducing glomerular hypertrophy than enalapril, other mechanisms in addition to the RAS are likely involved in hyperuricemia-induced glomerular hypertrophy.

Glomerular hypertrophy has been associated with the development of glomerulosclerosis [8]. In this regard, we found that rats made hyperuricemic for 6 months showed increased albuminuria and more glomerulosclerosis. These interesting data can only be viewed as provocative as we did not include rats receiving OA in which hyperuricemia was prevented (i.e., AP-treated) or blood pressurecontrolled (i.e., HCTZ-treated). Nevertheless, they suggest that glomerular hypertrophy in hyperuricemia may have important long-term consequences.

Several caveats should be made about the experimental protocol. First, when the study was performed, we were unaware that there is a diurnal variation of serum uric acid when animals are administered OA in their diet [13]. Uric acid levels tend to be higher in the early morn- 
ing after they have ingested the oxonate diet during the night, and uric acid levels tend to fall during the day [13]. Thus, it seems likely that the differences in uric acid levels between the OA-treated and control rats (which were often collected in late afternoon) might have been greater. Second, our studies were performed using an LS diet, as we have previously reported that the effects of hyperuricemia on blood pressure and renal structure are greatest under these dietary conditions [6]. While LS diets may be beneficial in many models by lowering systemic pressure, LS diets may also activate the RAS and can exacerbate some forms of renal injury such as that induced by cyclosporine [14]. Indeed, we recently reported that hyperuricemia increases renal renin content and accelerates renal injury in the LS diet/cyclosporine nephropathy model [14]. However, we have also found that hyperuricemic rats on a normal salt diet also have increased renin content [6]; preliminary studies suggest these rats also develop glomerular hypertrophy (data not shown). Finally, it is known that tail-cuff blood pressure measurements may also be variable; however, our rats were preconditioned (which reduces variability) and the technique used has been found to correlate closely with intra-arterial measurements [9].

In conclusion, hyperuricemia in the rat results in glomerular hypertrophy through a mechanism that is partially mediated by the RAS. While one must be cautious in extrapolating animal models to human disease, our studies provide a pathophysiological mechanism linking uric acid to renal injury and should stimulate clinical trials to address whether lowering uric acid may slow the progression of renal disease in man.

\section{Acknowledgements}

Support for this study was provided by an unrestricted grant from Merck, Inc., and from US Public Health Service Grant HL-68607.

\section{References}

1 Johnson RJ, Kivlighn SD, Kim YG, Suga S, Fogo AB: Reappraisal of the pathogenesis and consequences of hyperuricemia in hypertension, cardiovascular disease and renal disease. Am J Kidney Dis 1999;33:225-234.

2 Yü T, Berger L: Impaired renal function in gout: Its association with hypertensive vascular disease and intrinsic renal disease. Am J Med 1982;72:95-100.

3 Beck L: Requiem for gouty nephropathy. Kidney Int 1986;30:280-287.

4 Nickeleit V, Mihatsch MJ: Uric acid nephropathy and end-stage renal disease: Review of a non-disease. Nephrol Dial Transplant 1997;12: 1832-1838.

5 Wu X, Muzny DM, Lee CC, Caskey CT: Two independent mutational events in the loss of urate oxidase during hominoid evolution. J Mol Evol 1992;34:78-84.
6 Mazzali M, Hughes J, Kim YG, Jefferson J, Kang DH, Gordon KL, Lan HY, Kivlighn S, Johnson RJ: Elevated uric acid increases blood pressure in the rat by a novel crystal-independent mechanism. Hypertension 2001;35:11011106.

7 Mazzali M, Kanellis J, Han L, Feng L, Chen Q, Kang DH, Watanabe S, Nakagawa T, Lan HY, Johnson RJ: Hyperuricemia induces a primary renal arteriolopathy in rats by a blood pressureindependent mechanism. Am J Physiol Renal Physiol 2002;6:F991-F997.

8 Fogo AB: Glomerular hypertension, abnormal glomerular growth and progression of renal diseases. Kidney Int 2000;57:S15-S21.

9 Bunag RD, Butterfield J: Tail-cuff blood pressure measurement without external preheating in awake rats. Hypertension 1982;4:898-903.

10 Henry RJ, Sobel C, Kim J: A modified carbonate phosphotungstate method for the determination of uric acid and comparison with the spectrophotometric uricase method. Am J Clin Pathol 1957;28:152-160.
11 Kang DH, Hughes J, Mazzali M, George FS, Johnson RJ: Impaired angiogenesis in the remnant kidney model. II. Vascular endothelial growth factor administration reduces renal fibrosis and stabilizes renal function. J Am Soc Nephrol 2001;12:1448-1457.

12 Saito I, Saruta T, Kondo K, Nakamura R, Ogura T, Yamagami K, Ozawa Y, Kato E: Serum uric acid and the renin-angiotensin system in hypertension. J Am Geriatr Soc 1978;26:241247.

13 Newburger J, Combs AB, Hsu TF: Diurnal variation in serum uric acid of rats fed potassium oxonate. Drug Chem Toxicol 1978;1: 231-235.

14 Mazzali M, Kim YG, Suga S, Gordon KL, Kang DK, Jefferson JA, Hughes J, Kivlighn SD, Lan HY, Johnson RJ: Hyperuricemia exacerbates chronic cyclosporine nephropathy. Transplantation 2001;71:900-905. 